ten Signalwegen, etwa dem Notch- oder dem Wnt-Signalweg. Ein weiteres Ergebnis dieser Untersuchungen: die klonale Evolution ist bei der CLL zeitlich sehr gut zu verfolgen [u. a. Landau DA et al. Nature. 2015;526(7574):525-30]. „Im Unterschied zu vielen anderen Neoplasien hat die CLL typischerweise große Defekte von Chromosomen" erklärte Hallek. Del13q ist die häufigste, es gibt aber auch prognostisch günstige wie del11q und Trisomie 12. Damit beginne die Krankheitsentwicklung oft und in der Folge komme es dann zu weiteren, häufig auch prognostisch ungünstigen Mutationen wie z. B. in TP53.

Zum anderen wird dieser Therapieansatz greifbar, weil es extrem potente Substanzen zur CLL-Behandlung gibt, mit denen man auch klonale Veränderungen adressieren kann. Hallek berichtete exemplarisch von den bereits zugelassenen Optionen Idelalisib, einem PI3K- $\delta$-Inhibitor, und Ibrutinib, einem BTK-Hemmer, auf den auch Patienten mit del17p ansprechen. Eine weitere Substanz, die kurz vor der Zulassung stehe, sei der BCL-2-Antagonist Venetoclax. Er hemme den antiapoptotischen Effekt von BCL-2 und führe damit zu einer extrem eindrucksvollen klinischen Wirkung. „Gerade bei Patienten mit Rezidiv haben alle diese Substanzen sehr hohe Ansprechraten gezeigt", so Hallek.

\section{Mikromilieu unterschätzt?}

Alle drei Substanzen hemmen die Signalkaskade des B-Zell-Rezeptors und unterbrechen die proliferativen Signale der B-Zelle. Ergebnisse aus seiner Abteilung könnten Hallek zufolge aber das Verständnis der CLL-Pathogenese revolutionieren. Es sind Daten von Knock-out-Versuchen im Mausmodell mit der Tyrosinkinase LYN, die ganz oben in der B-Zell-Signalkaskade steht. Die Resultate ließen vermuten, so Hallek, dass Makrophagen eine wichtige Rolle spielen. Wenn diese fehlten, könne die Unterstützung für die CLL-Zelle nicht mehr hergestellt werden. „Ibrutinib und Idelalisib könnten vor allem deshalb wirken, weil sie das Mikromilieu der CLL völlig verändern. “Das könne auch klinisch abgeleitet werden, denn unter der Therapie mit diesen Substanzen komme es anders als unter den Antikörpern nicht zu einem schnellen Absterben der Leukämiezellen, sondern zu einer Umverteilung.

Bleibt die Frage, wie die Fülle neuer Möglichkeiten bei der CLL künftig eingesetzt wird. Halleks Antwort darauf: Die Optionen würden künftig in Kombination eingesetzt. In Untersuchungen der CLL-Studiengruppe werde dieser Ansatz bereits verfolgt, z. B. in der Phase-III-Studie CLL-14. Neben großen Phase-III-Studien würden verschiedene Kombinationen jetzt in kleinen Phase-II-Studien getestet. Hallek erklärte das praktische Vorgehen wie folgt: Zu Beginn müsse der Tumor reduziert werden, weil der Tumorzerfall aufgrund der starken Wirksamkeit der Substanzen so groß sei. Im Anschluss daran würden ein Jahr lang nur noch die Inhibitoren mit Antikörpern kombiniert. Und dann erfolge nur eine Therapie, wenn eine Resterkrankung nachweisbar sei. In diesem Vorgehen sieht Hallek die Zukunft: Anhand des Kurvenverlaufs werde abgeschätzt, wie die klonale Evolution erfolge und wie lange noch behandelt werden müsse, um auf sehr geringe Leukämiezellzahlen von z. B. $10^{-15} \mathrm{zu}$ kommen. Zeigt sich ein klonales Ereignis, also ein Resistenzmechanismus, wird neu sequenziert, die Therapie angepasst und der Abfall der Leukämiezellen überwacht.

Wichtig sei dies vor allem bei Patienten mit besonderem Risiko, etwa einer TP53-Mutation oder del17p und IGHV-Wildtyp.

\section{kurz notiert}

Liquid Biopsies: Es sind noch einige Hürden zu nehmen

Das Interesse am Nachweis von Tumorzellen und krebsspezifischer DNA im peripheren Blut wächst. Als einzige diagnostische Methode reichen "Liquid Biopsies" jedoch noch nicht aus.

Ziel der "Liquid Biopsies“ ist zunächst die frühzeitige Krebsdiagnose. Allerdings sind nach Ansicht von Klaus Pantel, Hamburg, vor einer routinemäßigen Anwendung noch einige Hürden zu überwinden. Zwar lassen sich Tumorzellen im Blut nachweisen, und ihre Zahl hatte in kleinen Studien bei Brust-, Darm- und Prostatakarzinom prognostisch Bedeutung: je höher, desto schlechter die Prognose. Die klinische Bedeutung sei jedoch unklar, so Pantel beim Krebskongress in Berlin. Bei Hirntumoren habe man zudem bisher angenommen, dass keine Krebszellen wegen der Blut-HirnSchranke im peripheren Blut auftauchen. Inzwischen wurden in einer Studie bei jedem fünften Patienten doch im Blut solche Zellen entdeckt. Das habe z. B. für die Transplantationsmedizin Bedeutung, wenn Spenderorgane von Hirntoten stammen, die an einem Hirntumor erkrankt waren. Dann würden möglicherweise Krebszellen mit übertragen.

Nach Berechnungen kann bei Krebskranken im peripheren Blut eine maligne Zelle unter einer bis 100 Millionen gesunden Zellen entdeckt werden. Ihre Halbwertszeit liegt zwischen nur einer und knapp zweieinhalb Stunden. Wie viele letztlich im Blut auftauchen, ist bei jeder Tumorart anders. Was die Analyse zudem weiter erschwert, ist die große genomische und phänotypische Heterogenität der Zellen in ein und demselben Patienten. Außerdem tauchen die Krebszellen nicht immer nur einzeln auf, sondern etwa in Clustern mit anderen Zellen. Schließlich wies Matthias Ebert, Mannheim, darauf hin, dass beim Nachweis von Zellen solider Tumoren im Blut zunächst unklar ist, ob sie den gesamten Primärtumor repräsentieren oder nur einen Teil.

Auch von der frei im Blut zirkulierenden DNA (cfDNA, cell free DNA) erhoffen sich Onkologen und Pathologen aussagekräftige Hinweise, die sich für Diagnostik und Therapieplanung nutzen lassen. Im Vergleich zum Tumorzellnachweis ist das Verfahren zwar näher an der klinischen Anwendung. Aber was sich da im Blut an genetischem Material nachweisen lässt, stammt nicht nur von Tumorzellen, sondern auch von gesunden Zellen, die zugrunde gegangen sind. Die Herausforderung ist, die DNA der verschiedenen Zellarten zuverlässig zu unterscheiden. Man muss sich deshalb an krebsspezifischen Mutationen orientieren. Wie Jung berichtete, gibt es seit 2014 ein von der Europäischen Arzneimittelagentur (EMA) zugelassener Test, mit dem sich EGFR-Mutationen in Tumorzellen aus dem Blut von Lungenkrebspatienten nachweisen lassen. Er darf demnach erst dann angewendet werden, wenn adäquates Gewebe für die Histologie nicht verfügbar ist.

Beim derzeitigen Forschungsstand plädiert Pantel für einen pragmatischen Ansatz, nämlich für die Diagnostik die Kombination aus pathohistologischer Untersuchung und "Liquid Biopsies" zu wählen Peter Leiner

\section{S3-Leitlinie: Palliative Chemotherapie beim Ösophaguskarzinom}

Die AWMF-S3-Leitlinie zum Ösophaguskarzinom ist die erste Leitlinie, in der zwischen Adenokarzinomen und Plattenepithelkarzinomen in der palliativen Chemotherapie unterschieden wird [AWMF-Registernr. 021/023OL]. Der Erhalt der Lebensqualität steht bei der Therapie im Vordergrund. Darauf verwies Udo Vanhoefer, Hamburg. 50 bis $60 \%$ aller Ösophaguskarzinome sind Plattenepithelkarzinome, der Anteil der Adenokarzinome ist mittlerweile auf über ein Drittel gestiegen. Trotz Verbesserungen sind die Überlebensaussichten beim Ösophaguskarzinom noch immer ungünstig: Die relative 5-Jahresüberlebensrate beträgt bei Männern derzeit $22 \%$, bei Frauen $24 \%$. Nur etwa jeder siebte Tumor wird in einem frühen (T1) Stadium diagnostiziert [Krebs in Deutschland 2011/2012. 10. Ausgabe. RKI und GEKID e.V]. 